Check for updates

Cite this: RSC Adv., 2019, 9, 13418

\title{
Effect of interfacial defects on the electronic properties of graphene/g-GaN heterostructures
}

\author{
Zhongxun Deng, (D) Xianhui Wang* and Jie Cui \\ To gain deep insights into their interactions, the effects of interfacial defects on the structural and electronic \\ properties of graphene/g-GaN heterostructures were investigated by using first-principles calculations. The \\ graphene/g-GaN- $\mathrm{V}_{\mathrm{Ga}}$ heterostructure maintains a p-type Schottky contact in the spin-up channel and the \\ Schottky barrier height $(\mathrm{SBH})$ is decreased to $0.332 \mathrm{eV}$, but there is not a metal/semiconductor contact in \\ the spin-down channel. However, the $n$-type $\mathrm{SBH}$ is negative for the graphene/g- $\mathrm{GaN}-\mathrm{V}_{\mathrm{N}}$ heterostructure, \\ indicating an ohmic contact. Furthermore, the SBH in the graphene/g-GaN heterostructure can be \\ effectively modulated by the interlayer distance. The research could provide a strategy for the \\ development and fabrication of efficient novel nanoelectronic devices.
}

Received 2nd March 2019

Accepted 17th April 2019

DOI: 10.1039/c9ra01576k

rsc.li/rsc-advances

show promise for application in optoelectronic and electronic

\section{Introduction}

Recently, a great number of two-dimensional (2D) materials as promising candidates for next-generation electronic devices have been discovered and studied due to their outstanding properties. ${ }^{1-8}$ Graphene, a 2D sp ${ }^{2}$-hybridized carbon monolayer, is known to have many fascinating electronic and quantum transport properties, such as massless Dirac fermions, high carrier mobility and an intriguing quantum Hall effect, which makes it a potential material in nanoelectronic and related devices., ${ }^{910}$ Unfortunately, the gapless nature of graphene hinders its application in electronic devices. ${ }^{11,12}$ Despite considerable investigations toward bandgap opening in graphene, most of these methods are difficult to execute or they significantly sacrifice its high carrier mobility.

Currently, vertical heterostructures based on 2D van der Waals (vdW) materials, which integrate the properties of their respective components with ideal properties, are considered a promising method to construct nanoelectronic and optoelectronic devices. ${ }^{13-18}$ Many 2D ultrathin hybrid graphenebased vdW heterostructures have been widely studied experimentally and theoretically, such as graphene/h-BN, ${ }^{19}$ graphene/ $\mathrm{BP},{ }^{20}$ and graphene/TMDs. ${ }^{21}$ These graphene-based vdW heterostructures show novel optoelectronic properties far beyond their individual components. Moreover, for vdW heterostructures, most of the 2D materials form ideal substrates for each other to preserve their intrinsic electronic properties due to the lack of dangling bonds and the weak electron coupling between sublayers. For example, GaN thin films on a graphene layer exhibit excellent optical and electric characteristics and

Shanxi Province Key Laboratory of Electrical Materials and Infiltration Technology, School of Materials Science and Engineering, Xi'an University of Technology, Xi'an 710048, Shaanxi, P. R. China.E-mail:xhwang693@xaut.edu.cn devices. $^{22}$ Tian and his colleagues reported that a hybrid graphene/GaN phototransistor with a relatively large $15.2 \mathrm{~mm}^{2}$ active area can reach $361 \mathrm{~mA} \mathrm{~W}^{-1}$ at $10 \mathrm{~V}$ with a response time of only $\sim 5 \mathrm{~ms}$, a much better performance than traditional GaN photodetectors. ${ }^{23}$ However, GaN in contact with flat graphene exhibits Schottky behavior. ${ }^{24}$ As we know, in a contact between metals and semiconductors the Schottky barrier is mainly contributed by the difference in Fermi level and associated charge transport. To date, in order to solve the problem of the contact barrier, many approaches have been developed to extend the possible applications of $2 \mathrm{D}$ heterostructures, for instance by changing interlayer distance, layer stacking or electric fields. ${ }^{25,26}$ There is a transition from an n-type to a Schottky type contact at the graphene/GaN interface when the interlayer distance decreases from 4.5 to $2.5 \AA{ }^{27}$ Moreover, a band gap of up to $334 \mathrm{meV}$ was obtained in graphene/ graphene/GaN when the perpendicular electric field reached $1 \mathrm{~V} \AA^{-1} .{ }^{28}$ Subsequently, understanding the interaction between $\mathrm{g}$-GaN and graphene, and engineering the interfacial states are crucial for future applications.

In this work, the effect of structural defects on the interfacial states of graphene/GaN vdW heterostructures was investigated using first-principles calculations. Then, the effect of the interlayer coupling on the electronic properties of graphene/gGaN heterostructures with defects was explored. For comparison, the effect of interlayer coupling on the electronic properties of graphene/g-GaN heterostructures without defects was also explored. The results exhibited that structural defects have a significant influence on the electronic properties of graphene/ $\mathrm{g}$-GaN vdW heterostructures. Moreover, tunable Schottky contacts and barrier can be achieved by modulating the interlayer coupling. The research is significant for the development and fabrication of efficient novel nanoelectronic devices. 


\section{Computational methods}

This work used density functional theory (DFT) with the projected augmented wave (PAW) method, as implemented within the Vienna Ab-Initio Simulation Package (VASP). ${ }^{29-32}$ The generalized gradient approximation was utilized as the exchange-correlation functional, which can reasonably predict the band gap of GaN. ${ }^{33}$ The vdW correction proposed by Grimme was included in our calculations due to its good description of long-range vdW interactions. ${ }^{34} \mathrm{~A}$ dipole correction is considered in the $z$ direction to remove the fake interaction between the dipole moments because the slab is not symmetric. The heterostructure calculations were performed using $5 \times 5 \times 1$ and $13 \times 13 \times 1$ Monkhorst-Pack $K$-point meshes for relaxation and electron static calculations, respectively. The Gaussian smearing method along the high symmetry line of $\Gamma(0,0,0)-M(0,1 / 2,0)-K(-1 / 3,2 / 3,0)-\Gamma(0,0,0)$ and a broadening of $0.2 \mathrm{eV}$ were adopted. The energy cutoff for plane-wave expansion was set to $450 \mathrm{eV}$. A large vacuum space of $20 \AA$ was added to avoid interaction between adjacent images. All atoms were allowed to be fully relaxed until the atomic Hellmann-Feynman forces were smaller than $0.01 \mathrm{eV} \AA^{-1}$ and the convergence criterion of energy in the self-consistency process was set to $10^{-5} \mathrm{eV}$. Visualizations of supercells and structures were attained with the software VESTA. ${ }^{35}$

The binding energy at the interface can be defined as

$$
E_{\mathrm{b}}=\left[E_{\mathrm{GaN} / \text { graphene }}-\left(E_{\mathrm{GaN}}+E_{\text {graphene }}\right)\right] / N_{\mathrm{C}},
$$

where $E_{\mathrm{b}}$ is the binding energy; $E_{\mathrm{GaN} / \text { graphene }}, E_{\mathrm{GaN}}$, and $E_{\text {graphene }}$ are the total energy of the graphene/g-GaN heterostructure, gGaN monolayer, and graphene monolayer, respectively; and $N_{\mathrm{C}}$ is the number of carbon atoms in the supercell. The system is stable for negative $E_{\mathrm{b}}$, and the smaller the $E_{\mathrm{b}}$ value is, the stronger the heterostructure binding is. To gain more insights into the bonding nature and interlayer interaction, the planeaveraged charge density difference $\Delta \rho$ is calculated as follows:

$$
\Delta \rho=\rho_{\mathrm{GaN} / \mathrm{graphene}}-\rho_{\mathrm{GaN}}-\rho_{\text {graphene }}
$$

where $\rho_{\mathrm{GaN} / \text { graphene, }}, \rho_{\mathrm{GaN}}$, and $\rho_{\text {graphene }}$ are the plane-averaged charge densities of the GaN/graphene heterostructure, GaN and graphene, respectively.

\section{Results and discussion}

\subsection{Structural properties}

Top views of the crystal structures of graphene/g-GaN without or with defects (Ga or $\mathrm{N}$ vacancies) are shown in Fig. 1. The calculated lattice constant of the free-standing graphene is $2.496 \AA\left(a_{g}=2.496 \AA\right)$, which is consistent with the experimental value. ${ }^{36}$ The optimized lattice constant of the GaN monolayer is $3.253 \AA\left(a_{\mathrm{GaN}}=3.253 \AA\right)$, which agrees well with previous reports. ${ }^{37}$ It is generally known that $(\sqrt{3} \times \sqrt{3}) R 30^{\circ}$ graphene can be easily generated on a $6 \mathrm{H}-\mathrm{SiC}$ substrate by epitaxial growth. ${ }^{38,39}$ Moreover, $3 \sqrt{3} a_{\mathrm{g}}=12.969 \AA$ for graphene and $4 a_{\mathrm{GaN}}=13.012 \AA$ for GaN. To minimize the lattice mismatch

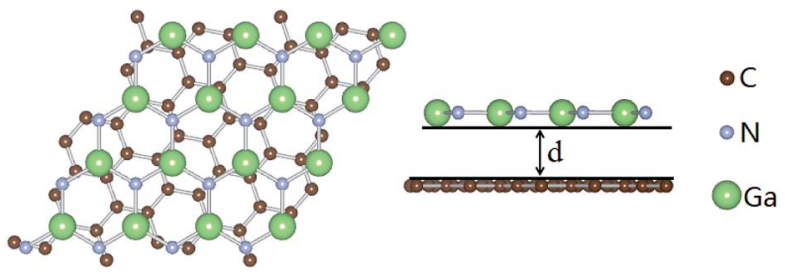

(a)

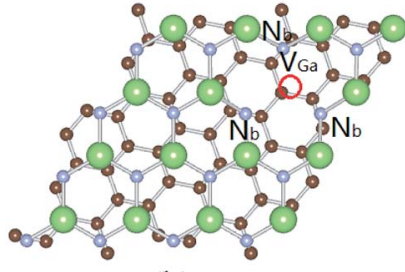

(b) (c)

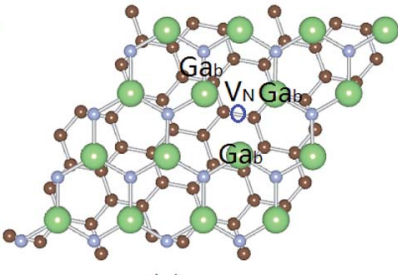

Fig. 1 A schematic illustration of the crystal structures of graphene/gGaN with or without defects: (a) graphene/g-GaN, (b) graphene/g$\mathrm{GaN}-\mathrm{V}_{\mathrm{Ga}}$, and (c) graphene/g-GaN- $\mathrm{V}_{\mathrm{N}}$.

between the stacking sheets, a $(\sqrt{3} \times \sqrt{3}) R 30^{\circ}$ cell is adopted as the unit cell of graphene, whose lattice constant is $3.323 \AA^{40}$ For all the heterostructures considered in this work, supercells consisting of $3 \times 3$ unit cells of graphene and $4 \times 4$ unit cells of GaN without or with defects are adopted, which leads to a small lattice mismatch of $0.3 \%$ in the supercell. In the established models, the lattice constant of GaN is fixed and the lattice constant of graphene is changed to adjust the heterostructures. The supercells are then fully relaxed for the atomic geometry.

The interlayer spacing $(d)$, bond lengths $\left(L_{\mathrm{C}-\mathrm{C}}\right.$ and $\left.L_{\mathrm{Ga}-\mathrm{N}}\right)$ and binding energy $\left(E_{\mathrm{b}}\right)$ for the considered structures are listed in Table 1. For g-GaN with $\mathrm{V}_{\mathrm{Ga}}$ and $\mathrm{V}_{\mathrm{N}}$, the interlayer spacing of the heterostructures are 3.287 and $3.225 \AA$, respectively, which are slightly smaller than that of the graphene/g-GaN heterostructure $(3.296 \AA)$. Moreover, their binding energies are 56 and $60 \mathrm{meV}$, which are the same order of magnitude as those of vdW crystals, such as $\mathrm{MoS}_{2} /$ graphene, ${ }^{41}$ black phosphorene/graphene, ${ }^{42}$ or arsenene/graphene. ${ }^{43}$

\subsection{Electronic properties}

The band structures of the graphene/g-GaN heterostructure without or with defects in its equilibrium configuration are shown in Fig. 2. From Fig. 2(a), it is evident that both the Dirac cone of graphene and the semiconducting nature of g-GaN are quite well preserved. The linear dispersion characteristic of

Table 1 Interlayer distance $(d)$, bond length $(L)$, and binding energy $\left(E_{\mathrm{b}}\right)$ of vdW heterostructures

\begin{tabular}{lcccc}
\hline System & $d(\AA)$ & $L_{\mathrm{C}-\mathrm{C}}(\AA)$ & $L_{\mathrm{Ga}-\mathrm{N}}(\AA)$ & $E_{\mathrm{b}}\left(\mathrm{meV} \mathrm{C}^{-1}\right)$ \\
\hline Graphene & & 1.422 & & \\
GaN & & & 1.880 & \\
Graphene/g-GaN & 3.296 & 1.422 & 1.880 & 54 \\
Graphene/g-GaN-V & 3.287 & 1.423 & 1.882 & 56 \\
Graphene/g-GaN-V & 3.225 & 1.421 & 1.881 & 60
\end{tabular}



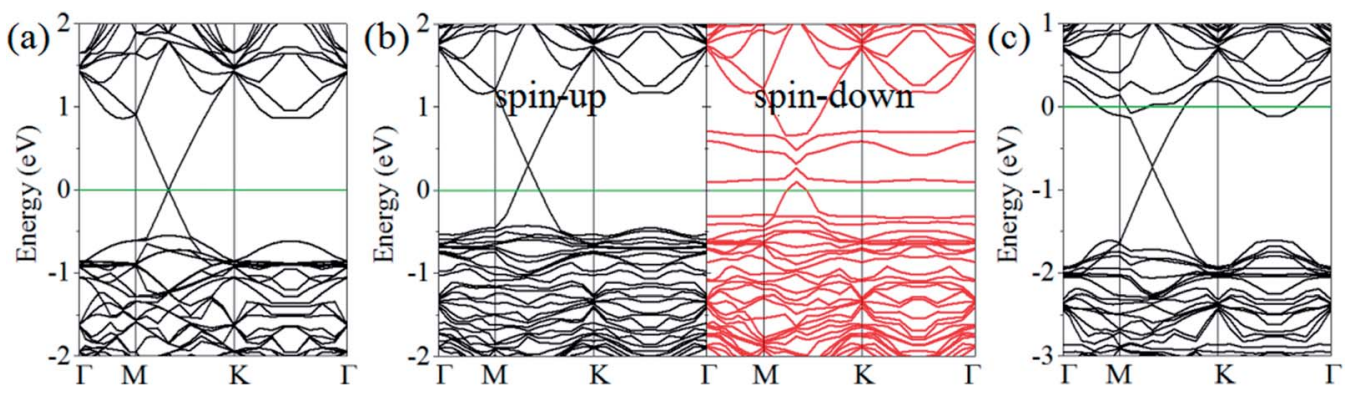

Fig. 2 The band structure of graphene/g-GaN with or without defects: (a) graphene/g-GaN, (b) graphene/g-GaN-V $\mathrm{Ga}_{\mathrm{G}}$ and (c) graphene/g-GaN- $\mathrm{V}_{\mathrm{N}}$.

graphene is retained in bands near the Fermi level. In addition, the Fermi level is located exactly at the Dirac cone, demonstrating that the effects of the interaction and charge transfer are vanishingly small. Compared with the band gap of $2.20 \mathrm{eV}$ for free-standing $\mathrm{GaN},{ }^{44,45}$ the band gap of $1.893 \mathrm{eV}$ for the $\mathrm{g}$ GaN sublayer decreases when in contact with graphene. Furthermore, the bandgap width depends on the $2 \mathrm{p}$ orbit of $\mathrm{N}$ atoms in the g-GaN sublayer (Fig. 3(a)). These results suggest that graphene could be a very promising material for the metal electrode, and it could enhance the contact performance while the electronic properties of g-GaN could be sustained. Then, a Schottky barrier could be formed between graphene and gGaN, which is similar to the coupling of graphene with BP or TMDs. Based on the Schottky-Mott rule, the corresponding Schottky barrier height (SBH) is determined by the energy levels of band edges in the semiconductor and Fermi levels in the metal. Here, the n-type $\operatorname{SBH}\left(\Phi_{\mathrm{B}, \mathrm{n}}\right)$ is defined as the energy difference between the conduction band minimum (CBM) of the g-GaN sublayer and the Dirac cone of the graphene sublayer, while the p-type $\mathrm{SBH}\left(\Phi_{\mathrm{B}, \mathrm{p}}\right)$ is defined as the energy difference between the Dirac cone of graphene and the valence band maximum (VBM) of the g-GaN sublayer. For the graphene/gGaN heterostructure, the Dirac cone is close to the VBM of the $\mathrm{g}$-GaN sublayer. The value of $\Phi_{\mathrm{B}, \mathrm{p}}$ is $0.449 \mathrm{eV}$, much smaller than the value of $1.444 \mathrm{eV}$ for $\Phi_{\mathrm{B}, \mathrm{n}}$. Hence, a p-type Schottky contact is formed at the graphene/g-GaN interface.

For the graphene $/ \mathrm{g}-\mathrm{GaN}-\mathrm{V}_{\mathrm{Ga}}$ heterostructure shown in Fig. 2(b), the semiconductor nature in the spin-up channel and the metallic character in the spin-down channel are quite well preserved for the $G a N-V_{G a}$ sublayer, while the Dirac cone is destroyed for the graphene sublayer. Moreover, the value of $\Phi_{\mathrm{B}, \mathrm{p}}$ is $0.332 \mathrm{eV}$, much smaller than the value of $1.706 \mathrm{eV}$ for $\Phi_{\mathrm{B}, \mathrm{n}}$ in the spin-up channel. In addition, dangling bonds are produced on $\mathrm{N}$ atoms around the vacancy, inducing defect states between the VBM and CBM in the spin-down channel (Fig. 3(b)). However, g-GaN- $\mathrm{V}_{\mathrm{N}}$ exhibits an n-type semiconductor character in the graphene/g-GaN- $\mathrm{V}_{\mathrm{N}}$ heterostructure (Fig. 2(c) and 3(c)) and the value of $\Phi_{\mathrm{B}, \mathrm{n}}$ is $-0.158 \mathrm{eV}$, much smaller than the value of $1.454 \mathrm{eV}$ for $\Phi_{\mathrm{B}, \mathrm{p}}$. Therefore, an ohmic contact is formed at the graphene $/ \mathrm{g}-\mathrm{GaN}-\mathrm{V}_{\mathrm{N}}$ heterostructure.

Fig. 4 presents the electrostatic potential of graphene/g-GaN with or without defects. In the graphene/g-GaN heterostructure, the graphene layer has a deeper electrostatic potential than that of g-GaN. The difference between them across graphene/g-GaN is large, indicating a strong electronic field across the heterostructure. When there are Ga vacancies in g-GaN, the difference increases, while it decreases for $\mathrm{g}$-GaN with $\mathrm{N}$ vacancies. Moreover, for the graphene/g-GaN and graphene/g-GaN- $\mathrm{V}_{\mathrm{Ga}}$ heterostructures, the charges are depleted on the graphene side, while they are accumulated on the GaN side (Fig. 4(d) and (e)). The charges are depleted on the $\mathrm{GaN}-\mathrm{V}_{\mathrm{N}}$ side, whereas they are accumulated on the graphene side for graphene/g-GaN- $\mathrm{V}_{\mathrm{N}}$ (Fig. 4(c)). The results of Bader charge population analysis show that the charge transfer from graphene to $\mathrm{g}-\mathrm{GaN}\left(\mathrm{g}-\mathrm{GaN}-\mathrm{V}_{\mathrm{Ga}}\right)$ is $0.027 e(0.059 e)$, while the charge transfer from $\mathrm{g}-\mathrm{GaN}-\mathrm{V}_{\mathrm{N}}$ to graphene is $0.051 e$.

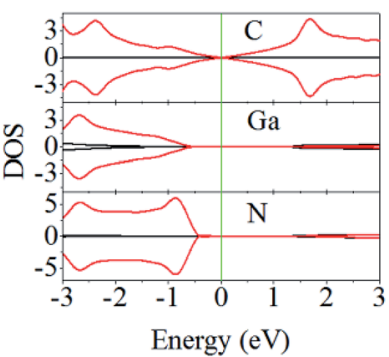

(a)

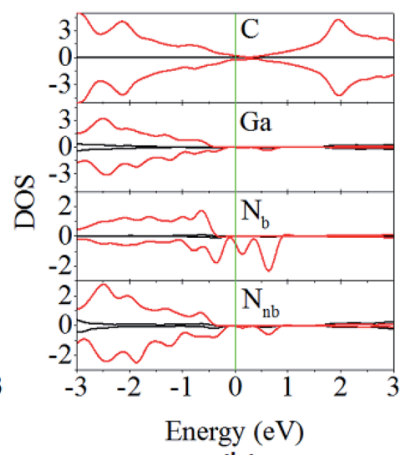

(b)

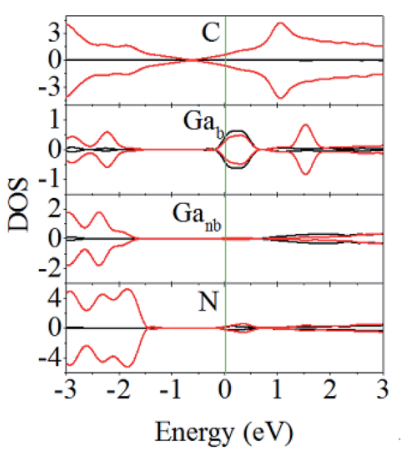

(c)

Fig. 3 The partial density of states (DOS) of graphene/g-GaN with or without defects: (a) graphene/g-GaN, (b) graphene/g-GaN-V $\mathrm{Ga}_{\text {a }}$ and (c) graphene/g-GaN- $V_{N}$. The black and red lines represent $\mathrm{s}$ and $\mathrm{p}$ orbitals of atoms, respectively. 


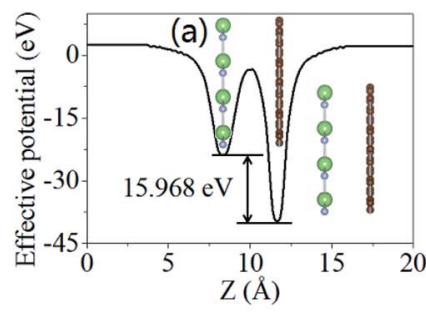

(d)

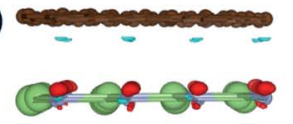

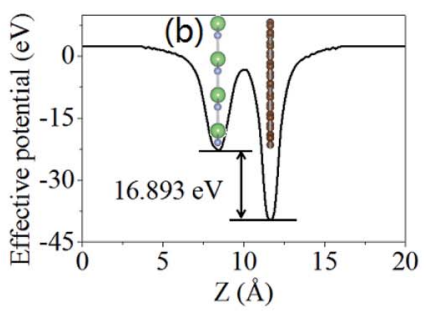

(e)

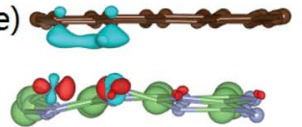

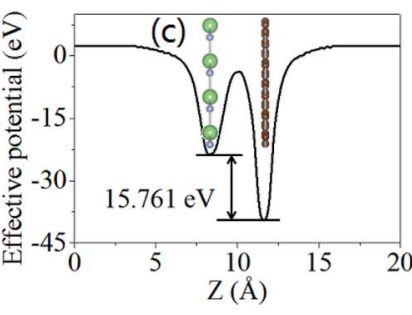

(f)

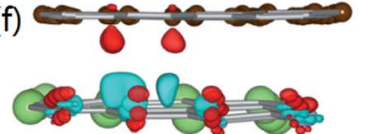

Fig. 4 Electrostatic potentials and difference charge density of graphene/g-GaN (a), graphene/g-GaN- $V_{G a}(b)$, and graphene/g-GaN- $V_{N}(c)$. The corresponding differences in charge density are shown in (d)-(f). The red and cyan isosurfaces represent the gain and depletion of electrons, respectively.

The above results show that the graphene/g-GaN, graphene/ $\mathrm{g}-\mathrm{GaN}-\mathrm{V}_{\mathrm{Ga}}$, and graphene/g-GaN- $\mathrm{V}_{\mathrm{N}}$ heterostructures are $\mathrm{p}$ type Schottky contact, p-type Schottky contact, and ohmic contact, respectively. Subsequently, for the design of graphene/GaN-based devices, structural defects might be desirable since they always improve the device performance. However, it is relatively well known that defect types are always uncontrolled during the fabrication of the g-GaN monolayer. Therefore, during fabrication of devices based on graphene/gGaN heterostructures, it is essential to prepare g-GaN with defects, especially $\mathrm{V}_{\mathrm{N}}$ defects.

\subsection{Effects of the interlayer coupling}

It is important to explore the effect of interlayer coupling on the electronic properties of the heterostructures. The electronic states of graphene/g-GaN with or without defects with different interlayer distances $(d)$ were calculated. For the graphene/g-GaN heterostructures, the results show that $\Phi_{\mathrm{B}, \mathrm{p}}$ is always smaller than $\Phi_{\mathrm{B}, \mathrm{n}}$, which indicates that the p-type Schottky contact remains despite the variations in $d$. As the interlayer distance decreases from $3.143 \AA$ to $2.969 \AA$, the values of $\Phi_{\mathrm{B}, \mathrm{p}}$ are negative, suggesting that the p-type Schottky contact turns to an ohmic contact (Fig. 5(a)). For the graphene/g-GaN-V $\mathrm{V}_{\mathrm{Ga}}$ heterostructure, the electronic structures are almost unchanged and the p-type Schottky contact remains despite the variations in $d$ (Fig. 5(b)). However, for the graphene/g-GaN- $\mathrm{V}_{\mathrm{N}}$ heterostructure, the values of $\Phi_{\mathrm{B}, \mathrm{n}}$ are changed, but always present as negative, which implies that an ohmic contact with different interlayer distances (Fig. 5(c)).

To better understand the variations in $\mathrm{SBH}$ with $d$ in graphene/g-GaN with or without defects, the $x y$-averaged electrostatic potential in the $z$ direction was calculated, as shown in Fig. 6. In the graphene/g-GaN heterostructure, the system is ptype Schottky contact when $d=3.629 \AA$ A. As $d$ decreases, the effect of interlayer coupling between the two sublayers becomes stronger, and more electrons move from graphene to g-GaN, which downward shifts the energy level of graphene close to the VBM of g-GaN, thereby decreasing the p-type SBH, resulting in a transition from a p-type Schottky contact to an ohmic contact at the interface (Fig. 6(a)). The same idea can also be applied to the graphene/g-GaN- $\mathrm{V}_{\mathrm{Ga}}$ heterostructure (Fig. 6(b)). As can be seen from Fig. 6(c), it is evident that more electrons are transferred from $\mathrm{GaN}-\mathrm{V}_{\mathrm{N}}$ to graphene as $d$ decreases from $3.611 \AA$ to $2.969 \AA$, which moves the energy level of graphene from the VBM down to the CBM of the $\mathrm{GaN}-\mathrm{V}_{\mathrm{N}}$ sublayer, eventually causing the transition from an ohmic contact to a nearly n-type Schottky contact at the interface.

In addition, it should be noted that, although it is still challenging to change the interlayer distance between graphene and g-GaN in the heterostructures experimentally, some research groups have been exploring how the interlayer distance of 2D layered-material systems could be tuned effectively in practice. For example, Tongay et al. experimentally demonstrated that the interlayer distance (coupling) of $\mathrm{WS}_{2} / \mathrm{MoS}_{2}$ heterostructures can be tuned by vacuum thermal annealing. ${ }^{46}$ Therefore, out-of-plane strain may be experimentally applied in a graphene/g-GaN heterostructure by means of vacuum thermal annealing in the near future, which will further affect the Schottky barrier
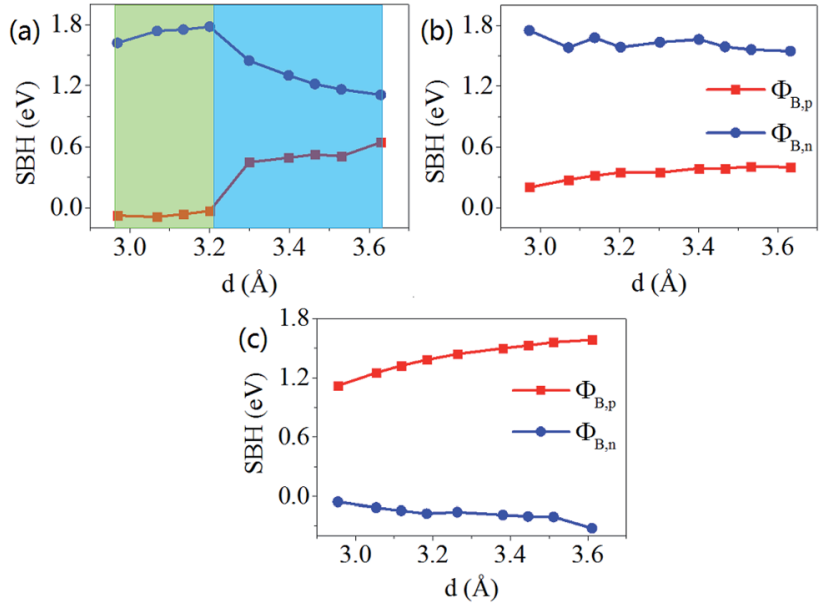

Fig. 5 The evolution of the Schottky barrier as a function of the interlayer distance for graphene/g-GaN (a), graphene/g-GaN-V $\mathrm{Ga}_{\text {a }}(\mathrm{b})$, and graphene/g-GaN- $V_{N}(c)$. 
(a)
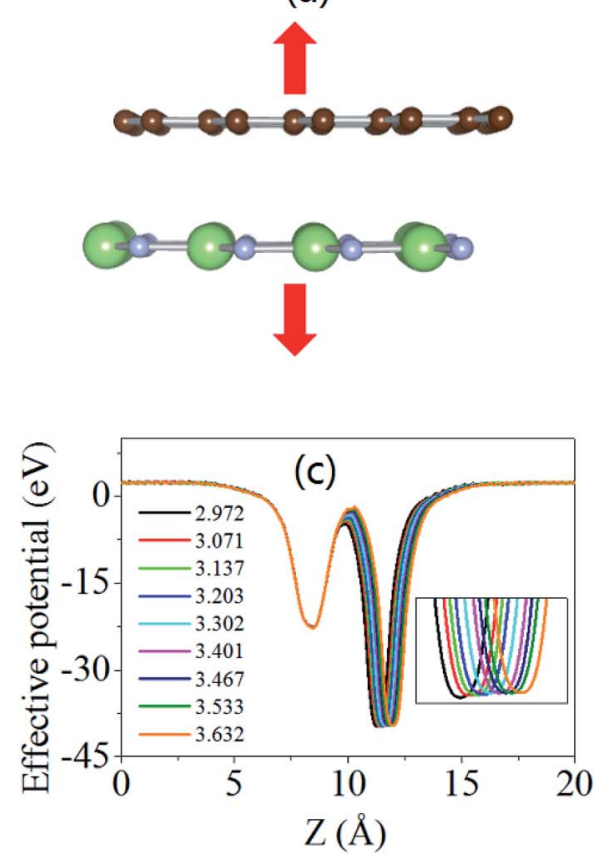
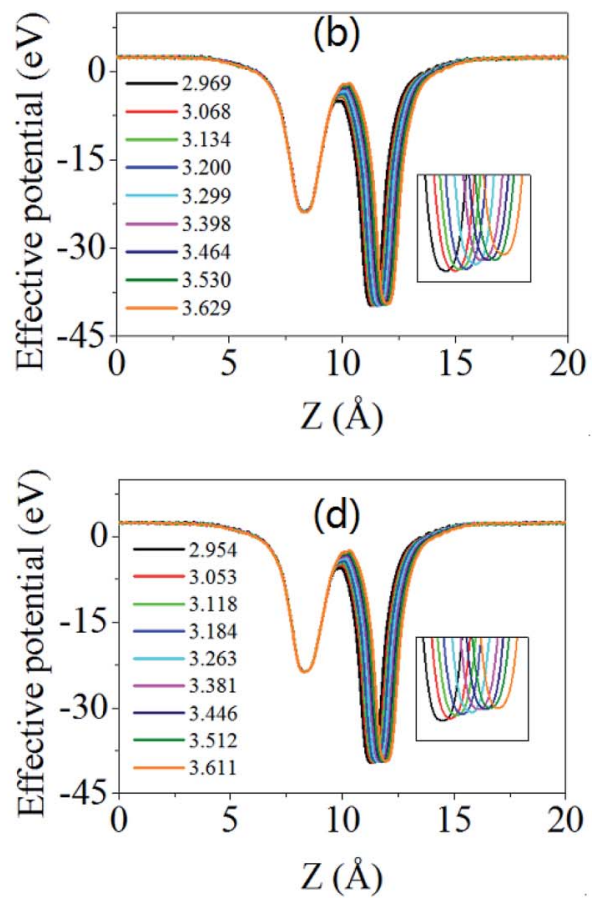

Fig. 6 A schematic diagram of vdW heterostructures with out-of-plane strain (a), and the electrostatic potential of graphene/g-GaN (b), graphene/g- $\mathrm{GaN}-\mathrm{V}_{\mathrm{Ga}}(\mathrm{c})$, and graphene/g- $\mathrm{GaN}-\mathrm{V}_{\mathrm{N}}$ with various interlayer distances (d).

and related electronic characteristics of graphene $/ \mathrm{g}-\mathrm{GaN}$ based electronic devices.

\section{Conclusions}

Systematic first-principles calculations were performed to understand the effects of structural imperfections on the electronic properties of graphene/g-GaN heterostructures. The findings can be summarized as follows:

(1) There is weak interaction between graphene and g-GaN with or without defects via vdW forces.

(2) The graphene/g-GaN heterostructure is a p-type Schottky contact with an SBH of $0.499 \mathrm{eV}$. The graphene $/ \mathrm{g}-\mathrm{GaN}-\mathrm{V}_{\mathrm{Ga}}$ heterostructure is still a p-type Schottky contact in the spin-up channel. However, the SBH is decreased to $0.332 \mathrm{eV}$. However, the graphene/g-GaN- $\mathrm{V}_{\mathrm{N}}$ heterostructure has become an ohmic contact with a negative value for $\Phi_{\mathrm{B}, \mathrm{n}}$.

(3) The large potential drop between graphene and g-GaN implies that a lower charge-injection barrier is formed at the interface. Moreover, the introduction of Ga vacancies in g-GaN can further lower the barrier at the graphene/g-GaN interface, resulting in better device performance.

(4) By varying the interlayer distance, the SBH in the graphene/g-GaN heterostructure can be effectively modulated. Interestingly, for the graphene/g-GaN- $\mathrm{V}_{\mathrm{N}}$ heterostructure, the ohmic contact remains with various interlayer distances.

\section{Conflicts of interest}

There are no conflicts to declare.

\section{Acknowledgements}

This research was supported by the National Natural Science Foundation of China (Grant No. 51274163 and 51605146), the Key Program of the National Natural Science Foundation of China (Grant No. U1502274), and the Key Science and Technology of Henan Province (Grant No. 172102410046).

\section{References}

1 K. S. Novoselov, A. K. Geim, S. V. Morozov, D. Jiang, Y. Zhang, S. V. Dubons, I. V. Grigorieva and A. A. Firsov, Science, 2004, 306, 666-669.

2 A. K. Geim and K. S. Novoselov, Nat. Mater., 2007, 6, 183.

3 K. Watanable, T. Taniguchi and H. Kanda, Nat. Mater., 2004, 3, 404 .

4 Q. L. Fang, X. M. Zhao, Y. H. Huang, K. W. Xu, T. Min, P. K. Chu and F. Ma, Phys. Chem. Chem. Phys., 2018, 20, 553. 5 B. Radisavljevic, A. Radenovic, J. Brivio, V. Giacometti and A. Kis, Nat. Nanotechnol., 2011, 6, 147.

6 Q. L. Fang, Y. H. Huang, Y. P. Miao, K. W. Xu, Y. Li and F. Ma, J. Phys. Chem. C, 2017, 121, 6605.

7 H. Liu, A. T. Neal and Z. Zhu, ACS Nano, 2014, 8, 4033.

8 R. Fei and Y. Li, Nano Lett., 2014, 14, 2884.

9 D. B. Farmer, H. Y. Chiu, Y. M. Lin, K. A. Jenkins, F. N. Xia and P. Avouris, Nano Lett., 2009, 9, 4474.

10 Y. B. Zhang, Y. W. Tan, H. L. Stormer and P. Kim, Nature, 2005, 438, 201.

11 M. Spinkle, D. Siegel, Y. Hu, J. Hicks, A. Tejeda, A. TablebIbrahimi, P. Le Fevre, F. Bertran, S. Vizzini, H. Enriquez, 
S. Chiang, P. Soukiassian, C. Berger, W. A. de Heer, A. Lanzara and E. H. Conrad, Phys. Rev. Lett., 2009, 103, 226803.

12 Y. Q. Wu, Y. M. Lin, A. A. Bol, K. A. Jenkins, F. N. Xia, D. B. Farmer, Y. Zhu and P. Avouris, Nature, 2011, 472, 7478.

13 Q. L. Fang, X. M. Zhao, Y. H. Huang, K. W. Xu, T. Min, P. K. Chu and F. Ma, J. Mater. Chem. C, 2018, 6, 4523.

14 Y. Q. Zhao, Q. R. Ma, B. Liu, Z. L. Yu, J. L. Yang and M. Q. Cai, Nanoscale, 2018, 10, 8677.

15 Y. Q. Zhao, X. Wang, B. Liu, Z. L. Yu, P. B. He, Q. Wang, M. Q. Cai and H. L. Yu, Org. Electron., 2018, 53, 50.

16 Z. L. Yu, Q. R. Ma, Y. Q. Zhao, B. Liu and M. Q. Cai, J. Phys. Chem. C, 2018, 122, 9275.

17 Q. L. Fang, X. M. Zhao, Y. H. Huang, K. W. Xu, T. Min and F. Ma, J. Mater. Chem. C, 2019, 7, 3607.

18 X. Z. Deng, Q. Q. Zhao, Y. Q. Zhao and M. Q. Cai, Curr. Appl. Phys., 2019, 19, 279.

19 B. Liu, Y. Q. Zhao, Z. L. Yu, L. Z. Wang and M. Q. Cai, J. Colloid Interface Sci., 2018, 513, 667.

20 J. Padilha, A. Fazzio and A. J. da Silva, Phys. Rev. Lett., 2015, 114, 066803.

21 H. V. Phuc, N. N. Hieu, B. D. Hoi, L. T. Phuong and C. V. Nguyen, Surf. Sci., 2018, 668, 23.

22 K. Chung, C. H. Lee and G. C. Yi, Science, 2010, 330, 655.

23 H. J. Tian, Q. L. Liu, A. Q. Hu, X. Y. He, Z. H. Hu and X. Guo, Opt. Express, 2018, 26, 5408.

24 H. J. Zhaong, Z. H. Liu, L. Shi, G. Z. Xu, Y. M. Fan, Z. L. Huang, J. F. Wang, G. Q. Ren and K. Xu, Appl. Phys. Lett., 2014, 104, 212101.

25 D. Lioyd, X. H. Liu, J. W. Christopher, L. Cantley, A. Wadehra, B. L. Kim, B. B. Goldberg, A. K. Swan and J. S. Bunch, Nano Lett., 2016, 16, 5836.

26 K. D. Pham, N. N. Hieu, H. V. Phuc, I. A. Fedorov, C. A. Duque, B. Amin and C. V. Nguyen, Appl. Phys. Lett., 2018, 113, 171605.

27 M. L. Sun, J. P. Chou, Q. Q. Ren, Y. M. Zhao, J. Yu and W. C. Tang, Appl. Phys. Lett., 2017, 110, 173105.

28 L. Huang, Q. Yue, J. Kang, Y. Li and J. B. Li, J. Phys.: Condens. Matter, 2014, 26, 295304.
29 G. Kresse and J. Hafner, Phys. Rev. B: Condens. Matter Mater. Phys., 1993, 47, 558.

30 G. Kresse and J. Hafner, Phys. Rev. B: Condens. Matter Mater. Phys., 1994, 49, 14251.

31 P. E. Blöchl, Phys. Rev. B: Condens. Matter Mater. Phys., 1994, 50, 17953.

32 J. P. Perdew, K. Burke and M. Ernzerhof, Phys. Rev. Lett., 1996, 77, 3865-3868.

33 Q. Chen, H. Hu, X. J. Chen and J. L. Wang, Appl. Phys. Lett., 2011, 98, 053102.

34 S. Grimme, J. Comput. Chem., 2006, 27, 1787.

35 K. Momma and F. Izumi, J. Appl. Crystallogr., 2008, 41, 653658.

36 L. Liu, P. Jewook, A. S. Devid, F. M. Kevin, W. C. Kendal, W. Deng, B. Leonardo, C. I. Juan, A. P. Li and G. Gu, Science, 2014, 343, 163.

37 K. S. Arunima and G. H. Richard, Appl. Phys. Lett., 2014, 105, 051604.

38 N. J. Huo, J. H. Yang, L. Huang, Z. M. Wei, S. S. Li, S. H. Wei and J. B. Li, Small, 2015, 11, 5430.

39 G. C. Constantinescu and N. D. M. Hine, Phys. Rev. B: Condens. Matter Mater. Phys., 2015, 91, 195416.

40 Y. X. Deng, Z. Luo, N. J. Conrad, H. Liu, Y. J. Gong, S. Najmaei, P. M. Ajayan, J. Lou, X. F. Xu and P. D. Ye, ACS Nano, 2014, 8, 8292.

41 T. Roy, M. Tosun, X. Cao, H. Fang, D. H. Lien, P. D. Zhao, Y. Z. Chen, Y. L. Chueh, J. Guo and A. Javey, ACS Nano, 2015, 114, 066803.

42 X. Lin, Y. Xu, A. A. Hakro, T. Hasan, R. Hao, B. L. Zhang and H. S. Chen, J. Mater. Chem. C, 2013, 1, 1618.

43 W. Hu, Z. Li and J. Yang, J. Chem. Phys., 2013, 139, 154704. 44 J. J. Guo, Z. P. Zhou, T. X. Wang, Z. S. Lu, Z. X. Yang and C. Liu, Curr. Appl. Phys., 2017, 17, 1714.

45 M. L. Sun, J. P. Chou, Q. Q. Ren, Y. M. Zhao, J. Yu and W. C. Tang, Appl. Phys. Lett., 2017, 110, 173105.

46 S. Tongay, W. Fan, J. Kang, J. Park, U. Koldemir, J. Suh, D. S. Narang, K. Liu, J. Ji, J. Li, R. Sinclair and J. Wu, Nano Lett., 2014, 12, 3185. 